\title{
Complete radiological response following subtotal resection in three glioblastoma patients under treatment with Tumor Treating Fields
}

\author{
ALMUTH FRIEDERIKE KESSLER ${ }^{1}$, THOMAS LINSENMANN ${ }^{1}$, THOMAS WESTERMAIER ${ }^{1}$, \\ WANJA WOLBER ${ }^{1}$, JUDITH WEILAND ${ }^{1}$, CAMELIA-MARIA MONORANU ${ }^{2}$, MARIA BREUN ${ }^{1}$, \\ CARSTEN HAGEMANN $^{1}$, RALF-INGO ERNESTUS ${ }^{1}$ and MARIO LÖHR ${ }^{1}$ \\ ${ }^{1}$ Department of Neurosurgery, University Hospital of Würzburg; ${ }^{2}$ Department of Neuropathology, \\ Institute of Pathology, University of Würzburg, Würzburg 97080, Germany
}

Received May 15, 2019; Accepted August 30, 2019

DOI: $10.3892 /$ ol.2019.11110

\begin{abstract}
Glioblastoma multiforme (GBM) treatment consists of surgery, radiotherapy and chemotherapy with Temozolomide (TMZ). After subtotal resection (STR), residual tumors rarely undergo spontaneous regression. Overall survival (OS) and progression-free survival (PFS) are reduced when compared with gross total resection. There is evidence that adding Tumor Treating Fields (TTFields) to standard management may lead to a significant increase in PFS and OS. In 2015 and 2016, STR was performed in 27 patients with GBM. Of these, four subsequently received TTFields therapy in addition to chemotherapy. The present study presents a series of three patients with GBM (44-54 years; isocitrate dehydrogenase wild-type, methylated O6-methylguanine-DNA methyltransferase promoter) that were treated with radiochemotherapy and TTFields after STR. Therapy with TTFields started concomitantly to TMZ following radiotherapy and was maintained for 14, 24 and 37 months. TTFields were used as monotherapy in one case, as TMZ treatment had to be stopped due to toxicity for 1 month. In all patients, TTFields therapy was well tolerated at high compliance levels, resulting in complete response (CR) after 4, 5 and 7 months, respectively. Two patients remain tumor-free at
\end{abstract}

Correspondence to: Dr Almuth Friederike Kessler, Department of Neurosurgery, University Hospital of Würzburg, 11 Josef-Schneider Street, Würzburg 97080, Germany

E-mail: kessler_a1@ukw.de

Abbreviations: EOR, extent of resection; GBM, glioblastoma multiforme; GTR, gross total resection; IDH, isocitrate dehydrogenase; KPS, Karnofsky performance score; MGMT, O6-methylguanine-DNA methyltransferase; OS, overall survival; PFS, progression-free survival; QoL, quality of life; STR, subtotal resection, TMZ, Temozolomide; TTFields, Tumor Treating Fields; WT, wild-type

Key words: complete response, incomplete resection, glioblastoma multiforme, Tumor Treating Fields, subtotal resection
16 and 40 months after STR. One patient exhibited multifocal recurrence 11 months after the beginning of TTFields treatment but remains alive, presenting a mild neurological decline 24 months after starting TTFields. All three presented patients gave written informed consent for their data to be published. In conclusion, the current report detailed three patients with GBM who underwent STR and were subsequently treated with TMZ and TTFields. TTFields treatment was tolerated well and was applied accurately and with high compliance by these patients, which may have contributed to the complete response. Four of the 27 patients treated with STR received additional TTFields treatment. Three of these 4 showed a CR, while a CR was observed only 2 of the remaining 23 patients without TTFields. The current series supports the effects in clinical practice, as demonstrated in recent clinical trials. The results also demonstrated that adjuvant TTFields therapy can structurally affect residual tumors after STR.

\section{Introduction}

Subtotal resection (STR) of the highly aggressive primary brain tumor glioblastoma multiforme (GBM) has been shown to significantly decrease the progression-free (PFS) and overall survival (OS) compared to gross total resection (GTR) (1). Various GBM trials have confirmed that an extent of resection (EOR) of $78 \%$ improves patients' outcome (2). Survival rates further increased when EOR rates of 96-100\% can be achieved. This is true not only for newly diagnosed GBM but also for recurrent $\operatorname{GBM}(3,4)$. In the latter, a cutoff of $80 \%$ EOR improved patients' outcome in the second line setting. However, even after repeated multimodal treatment, median survival is limited to approximately 20 months, which emphasizes the need for new treatment options (5).

Tumor Treating Fields (TTFields) are a local, non-invasive modality adjunct to first or second line therapy, which are delivered through transducer arrays placed on the shaved scalp and generated by a portable device. TTFields are alternating electric fields of intermediate frequency $(100-300 \mathrm{kHz}$, $200 \mathrm{kHz}$ for GBM) and low intensity (1-3 V/cm), which interfere with processes of mitosis to stop or slow down cell 
division and eventually induce cell death (6). The phase III trial EF-14 demonstrated that adding TTFields to standard chemotherapy significantly improved PFS and OS in patients with newly diagnosed GBM by 2.7 and 4.9 months, respectively (7). The compliance of the therapy is of importance as a post-hoc analysis of the EF-14 study revealed that higher compliance was associated with longer PFS and OS (8). The OS benefit of TTFields was independent of gender, O-6-methylguanine-DNA methyltransferase (MGMT) methylation status, age, region, performance status (KPS) and EOR (8) defined by MRI criteria as well as tumor progression.

Although complete radiological response (CR) was seen occasionally in the subgroup analysis of the EF-14 trial, no details, however, have been reported in literature about the clinical course of patients with residual tumors under TTFields treatment.

We report on three patients who showed complete radiologic response after subtotal resection of GBM under multimodal treatment including TTFields.

\section{Case report}

GBM patient characteristics following STR treatment in 2015 and 2016. Between 2015 and 2016, 27 patients received STR at our institution. Of the 27 STR-patients, 4 were treated with TTFields in addition to chemotherapy. Here, STR was defined as any residual contrast enhancing lesion. Three out of those 4 patients $(75 \% ; 3 / 4)$ displayed a CR, defined as no detectable contrast enhancement in the follow up after initial STR, while one patient remained radiologically stable for over 10 months before progression, which was treated by re-irradiation. Seventeen of the 23 patients without TTFields treatment developed early recurrence within 2 to 4 months while being on first-line standard therapy. Two patients were lost to follow up. One patient stayed radiologically stable for 8 months but then suffered from recurrent seizures, accompanied by a declining general condition and died after a short time in palliative care. One patient has remained stable for 10 months to-date. Only two of 23 patients who had not received TTFields had a CR in contrast enhancement after 6 and 15 months (8.7\%; 2/23).

All tumors were histologically assessed and graded on formaline fixed and paraffin embedded tissue sections by an experienced neuropathologist according to the 2016 criteria of the World Health Organisation. The IDH status was examined by immunohistochemistry using the antibody against IDH1 R132H and completed by a sequence analysis of IDH1 and IDH2 gene.

For determination of extent of resection, tumor progression as well as radiological responses to therapies RANO criteria were applied (9), which include diverse MRI criteria and clinical data as well. Observation period was closed in March 2018.

This study was carried out in accordance with the recommendations of the Declaration of Helsinky, in accordance with which all three presented subjects gave written informed consent for their data to be published.

Clinical course of patient one. Patient one (female, 54 years, KPS $80 \%$ ) presented with recurrent speech impairment. A $\mathrm{CT}$ scan revealed a left frontotemporal lesion with perifocal edema. MRI revealed an irregular contrast enhancing tumor highly susceptible for GBM, located in the frontal operculum and in close contact to the caudate nucleus and anterior crus of the internal capsule with moderate compression of the lateral ventricle and midline shift. After 5-ALA- and ultrasound-guided resection, the early postoperative MRI showed remaining tumor tissue in the rostral and dorsolateral marginal zones of the resection cavity, confirming a STR (Fig. 1A). Apart from a slight dysphasia, no neurological abnormalities were recorded after surgery. The histological diagnosis confirmed GBM WHO Grade IV, with methylated MGMT promoter, IDH1/2 wild-type and sustained nuclear expression of ATRX. Concomitant radiochemotherapy was administered (53.4 Gy/Temozolomide (TMZ) with $75 \mathrm{mg} / \mathrm{m}^{2}$ daily). A follow-up MRI at 3 months postoperatively and at 2 weeks after radiation termination showed a new contrast enhancement at the rostral margin of the left ventricle in an area initially suspected as non-contrast enhancing tumor. This new contrast enhancement was interpreted as pseudoprogression, while the postoperative residual tumor was defined as stable. The adjuvant chemotherapy with TMZ according to the Stupp protocol (10) was initiated 4 weeks after termination of radiochemotherapy. Application of TTFields was started at the same time. The patient was in a good general condition during the first cycle of TMZ, but suffered from persisting speech impairment. Due to leukocytopenia, the third cycle of TMZ was delayed for a month while TTFields therapy was applied continuously. Speech impairment improved, and the next follow-up MRI 4 months after surgery indicated reduced contrast enhancement of the pseudoprogression as well as the residual tumor. After termination of 6 cycles of TMZ, the patient continued with TTFields treatment at a median compliance rate of $92 \%$ [range: $88-96 \%$ ]. The MRI at five months after TTFields start did not show any contrast enhancement indicating a CR according to the RANO criteria (9) in both regions, residual tumor and pseudoprogression as well. The patient's status remained stable for 7 months (Fig. 1B) before multifocal tumor recurrence occurred. Since resection was not feasible, the patient was treated with re-irradiation and further 6 cycles of TMZ. TTFields was discontinued due to patient's decision at 12 months after initiation for 23 days. Afterwards, the patient resumed TTFields treatment, which is still ongoing for 24 months in total. The patient presents a mild decline of neurological functions due to slow but ongoing tumor growth.

Clinical course of patient two. Patient two (female, 46 years, KPS $90 \%$ ) displayed a right-sided hemiparesis. Her MRI revealed a left parietal lesion infiltrating the post-central gyrus. After STR contrast enhancement was still discernible at the resection margins $24 \mathrm{~h}$ after surgery (Fig. 2A). Histopathology confirmed GBM (methylated MGMT promoter, IDH1/2 WT, sustained nuclear expression of ATRX). Postoperative irradiation up to 61.2 Gy administered with concomitant daily TMZ $\left(75 \mathrm{mg} / \mathrm{m}^{2}\right)$ was well tolerated. Six cycles of adjuvant TMZ therapy were administered concomitantly with TTFields, which were applied for 14 months at compliance rate of above $90 \%$. Four months after initiating TMZ + TTFields, MRI revealed a CR of the residual tumor (Fig. 2B). After a follow-up period of 16 months, MRI did not show any signs of tumor recurrence. 
Clinical course of patient three. Patient three (male, 44 years, KPS $100 \%$ ) presented with seizures. The MRI showed a right frontal lesion involving parts of the corpus callosum. The FET-PET scan revealed a maximal tumor-to-background ratio (TBR) in the right anterior middle frontal gyrus. Early postoperative MRI showed residual tumor tissue at the margins of the resection cavity. Histopathology confirmed anaplastic astrocytoma WHO grade III. Subsequent irradiation therapy was administered up to $61.2 \mathrm{~Gy}$ with concomitant daily TMZ $\left(75 \mathrm{mg} / \mathrm{m}^{2}\right)$. After 4 cycles of adjuvant TMZ, a follow-up MRI revealed a progression of the contrast enhancing lesion at the inferior margin of the resection cavity and the corpus callosum, which was identified as a true tumor recurrence by a FET-PET scan. After repeated surgery, the early postoperative MRI presented a contrast enhancing tumor residue at the inferior margin of the resection cavity (Fig. 3A), extending into the genu of the corpus callosum (Fig. 3B) due to the subtotal resection. Histopathological workup revealed GBM WHO grade IV (methylated MGMT promoter, IDH1 R132H mutation). Due to the presence of methylated MGMT-promoter, TMZ therapy was resumed for another 6 cycles. In addition, TTFields treatment was initiated at cycle 4. During the first period of the TTFields treatment, the patient started at a compliance rate of $\sim 75 \%$. Interestingly, 6 months after surgery, the cranial MRI displayed a distinct regression of the contrast enhancement in the corpus callosum. Another 3 months later, no contrast enhancing tumor residues were discernible, which continued for 40 months after the second resection and until the end of the follow-up period (Fig. 3C and 3D). Simultaneously, the patient reported a high quality of life (QoL) and active participation in his Martial Arts training sessions as well as travel activities to foreign countries. Although suffering from psoriasis of the arms, legs and scalp, being treated with urea-based lotion and intermittendly with cortisol-based lotion, there were no adverse events besides mild skin irritation, no seizures or neurological abnormalities. The patient is working full-time and is permanently using the TTFields therapy with an average compliance of around $60 \%$ before terminating TTFields application after 37 and 40 months after STR up to the end of observation period.

\section{Discussion}

We present a series of three GBM patients presenting CR after STR according to the RANO criteria. All of these patients received adjuvant first line radio-chemotherapy and additional TTFields treatment. From 2015 to 2016, a total of 27 GBM patients underwent STR in our hospital. Four GBM patients received STR and were treated with TTFields. CRs were observed in three of these four subtotal resected GBM patients. Only 2 out of 23 GBM patients (8.7\%) being treated solely with standard radiochemotherapy after STR showed complete radiologic responses. This suggests a better radiologic response after STR by the addition of TTFields therapy.

The presented patients tolerated the TTFields treatment well for an average of 25 months and reported a good QoL. This is in line with the recently published QoL data from the EF-14 study that showed that health-related QoL during TTFields treatment was not impaired compared to the trial's control arm in all prespecified parameters, except for the complaint

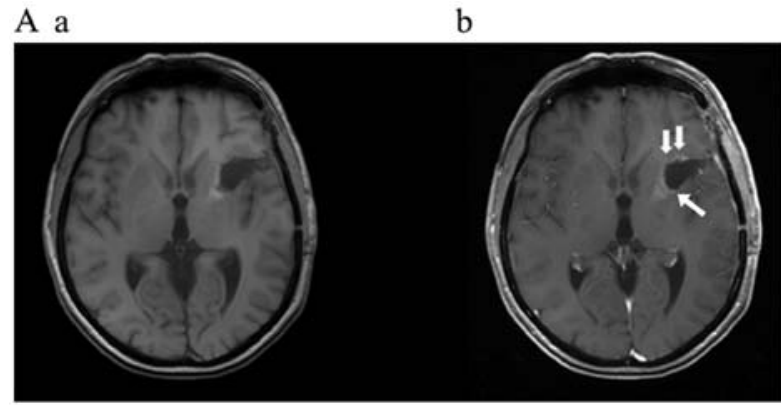

$\mathrm{B}$ a b

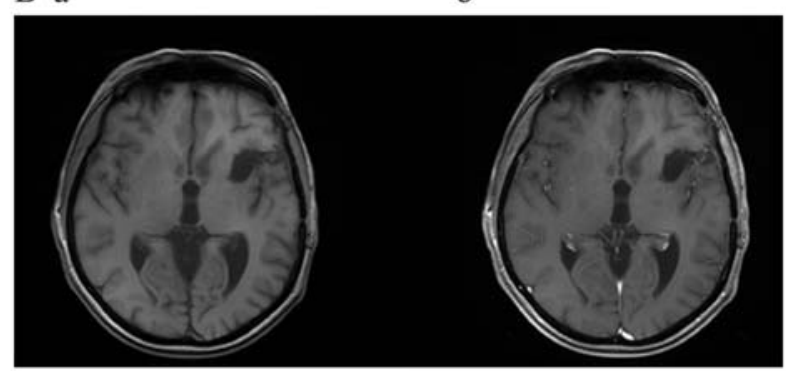

Figure 1. Axial T1-weighted MRI [native (Aa/Ab)] and contrast enhanced $(\mathrm{Ba} / \mathrm{Bb})$ images of patient one (female; 54 years old). (A) Incomplete resection was exhibited at $24 \mathrm{~h}$ post-surgery. (B) Complete radiological response according to rapid assessment in neuro-oncology criteria under dual therapy with TMZ and TTFields was demonstrated at 14 months. Arrows indicate residual contrast-enhancing tumor after STR. TMZ, Temozolomide; TTFields, Tumor Treating Fields.
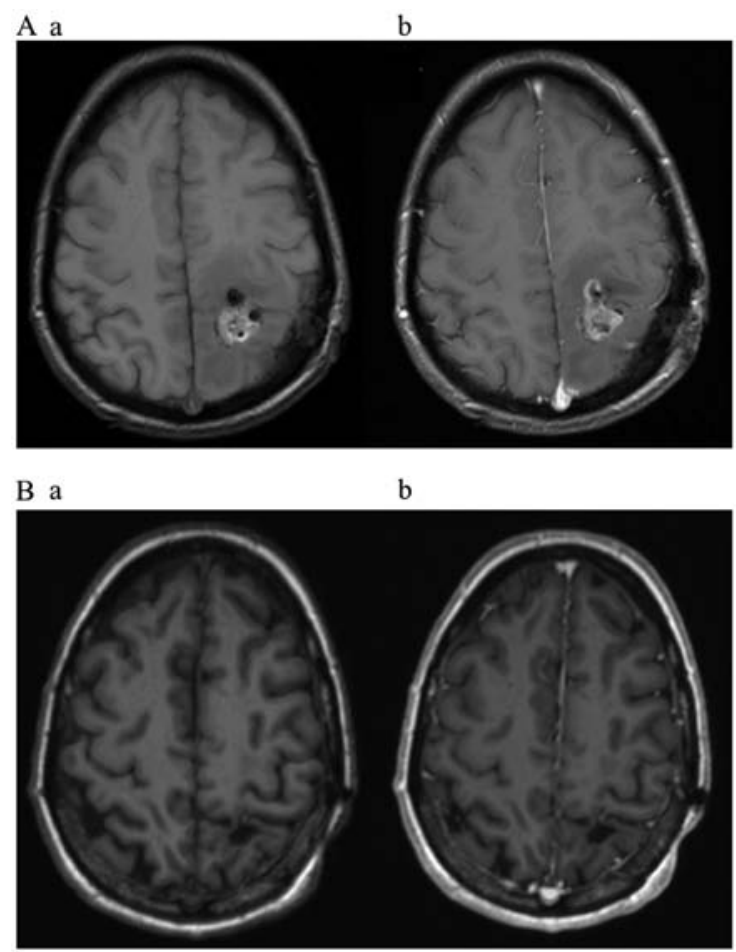

Figure 2. Axial T1-weighted MRI [native $(\mathrm{Aa} / \mathrm{Ab})$ ] and contrast enhanced $(\mathrm{Ba} / \mathrm{Bb})$ images of patient two (female; 46 years old). (A) Incomplete tumor resection is indicated at $24 \mathrm{~h}$ post-surgery. (B) Axial T1-weighted MRI exhibiting complete response 6 months after surgery and 4 months after the initiation of TTFields treatment. TTFields, Tumor Treating Fields.

of itchy skin (11). Applicability, tolerability and safety have already been shown in several previous studies $(6,7,12,13)$. In 
A a

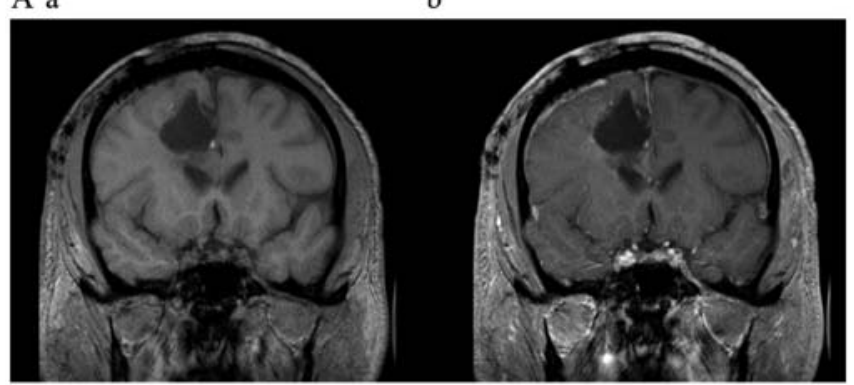

B a

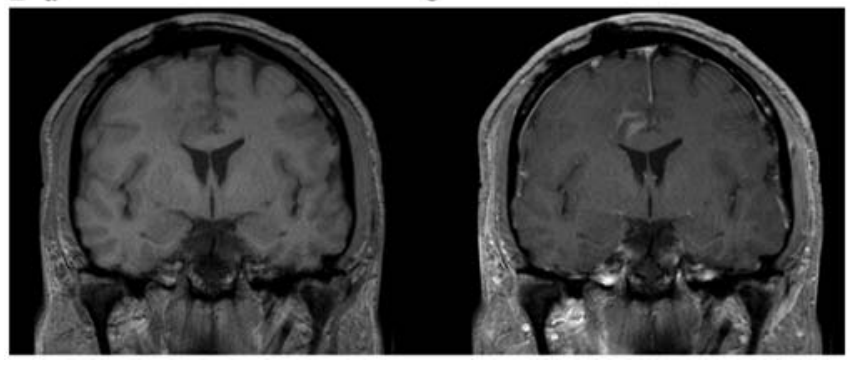

$\mathrm{Ca}$

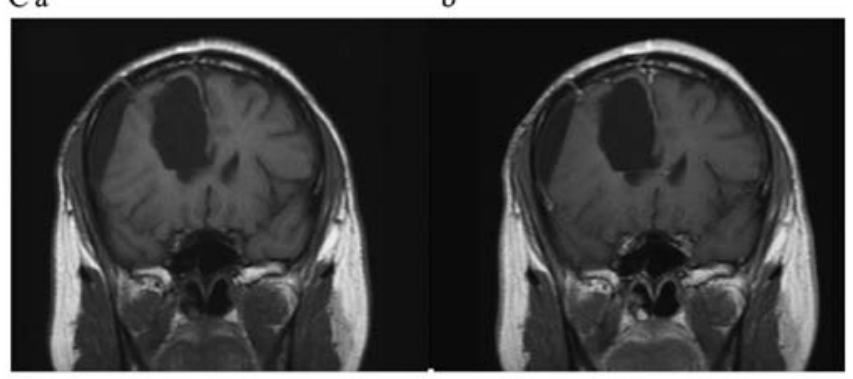

Da

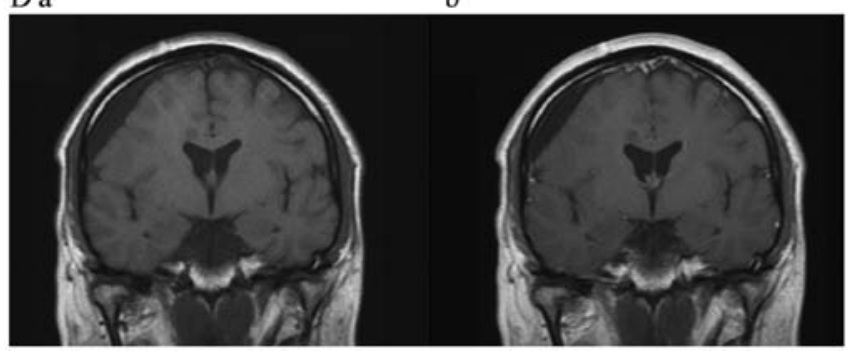

Figure 3. Coronal MRI of patient three (male; 44 years old) before $(\mathrm{Aa} / \mathrm{Ba} / \mathrm{Ca} / \mathrm{Da})$ and after $(\mathrm{Ab} / \mathrm{Bb} / \mathrm{Cb} / \mathrm{Db})$ gadolinium application. Early post-surgery MRI (48 $\mathrm{h}$ after surgery) was completed following the second STR with contrast enhancement in the marginal zone of the resection cavity (A) extending to the corpus callosum (B) (C) Images obtained from the same patient after another 6 cycles of Temozolomide and (D) 37 months of Tumor Treating Fields treatment (35 months as monotherapy). The patients presents complete response of the postoperative tumor residue 40 months after STR. STR, subtotal resection.

fact, patient 3 is still actively working in his job as a computer scientist, more than three years after the first diagnosis of an anaplastic astrocytoma. All patients participate regularly in recreational activities. The two female patients reached an average compliance rate above $90 \%$ during the entire treatment period. High compliance has been associated with better OS and PFS outcomes and may have contributed to their individual outcome (8). However, even compliance above a threshold of 50-60\% revealed significantly improved overall survival compared to the control group in a subgroup analysis of the EF-14 trial (8). Better compliance rates resulted in a further significant OS increase. Even though patient 3 has a very active lifestyle, he tolerated TTFields well enough to reach a compliance of over $60 \%$ throughout a 3 -year treatment period.

This is even more remarkable as this patient has been suffering from Psoriasis, which bears the risk of more severe skin reactions and infections induced by minor skin injury or irritations, the latter being one major side effect of TTFields. Prescribing this patient a skin irritating therapy was a matter of concern in his particular situation. This case shows that this patient with a skin disorder may well tolerate TTFields treatment with a good quality of life and no severe skin reactions to the therapy.

Furthermore, psoriasis, as seen in the third patient, might additionally be a prognosis-limiting factor. Some evidence indicates that there may be a negative association between brain tumors and psoriasis as well as other autoimmune diseases as chronic rheumatic heart disease, multiple sclerosis, and rheumatoid arthritis $(14,15)$. Although the third patient accumulated several positive prognostic factors as his high KPS of $100 \%$, methylated MGMT, mutated IDH1 and young age (44 years), the fast progression to a secondary GBM under ongoing TMZ therapy might have resulted from the confirmed psoriasis in combination with a STR $(16,17)$. But despite of the clinical course and the potentially negative prognostic factors, the patient presented a CR after the second STR which has previously been shown to reduce PFS and OS even in relapsing GBM (18). The second STR was followed by TMZ again, but this time accompanied by TTFields, suggesting that TTFields may have decisively contributed to this favorable clinical and radiological course.

The CRs of all three patients were seen after 4, 5 and 7 months, which is in line with the observation of a median 5.2 months in the responder patients in the EF-11 trial (19). All three patients had a complete response and are still alive in spite of their poor prognosis especially due to the extent of resection, but also their comorbidity and molecular profiles (20-22).

The observations of this report are supported by the subgroup analysis of the EF-14 trial. It demonstrated that the subgroup undergoing STR compared to GTR showed similar OS response when TTFields were added to their treatment regimen. OS in both groups exceeded 21 months from randomization (+4.1 months for GTR patients vs. +6.3 months for STR patients, HR: 0.7 vs. 0.56, respectively) (7).

In conclusion, adding TTFields to adjuvant chemotherapy is a valuable treatment option to improve clinical and radiological outcome after STR in both newly diagnosed and recurrent GBM. There are limitations due to the small sample size and the homogenous molecular-pathological profile. Therefore, these data should be further investigated. However, these observations in individual cases in clinical practice support the findings of the clinical trial EF-14. Thus, TTFields therapy can be recommended to all eligible GBM patients.

\section{Acknowledgements}

The authors would like to thank Miss Sandra Grebsattel (Department of Neurosurgery, Würzburg University Hospital) for her assistance with patient documentation. 


\section{Funding}

The current study was funded by the German Research Foundation (DFG) and the University of Wuerzburg in the funding program Open Access Publishing.

\section{Availability of data and materials}

The datasets used and/or analyzed are available from the corresponding author on reasonable request.

\section{Authors' contributions}

MB, TL, JW and WW reviewed patient clinical history, reviewed the literature and prepared the manuscript. $\mathrm{CH}$ and RIE reviewed the literature and revised the manuscript. $\mathrm{CH}$ contributed to conception and design of the study. RIE gave advice for data interpretation. AFK and CMM reviewed patient pathology and the manuscript. TW and ML reviewed the manuscript and MRI scans. AFK identified the patient, reviewed patient clinical history, performed a literature search, and prepared and edited the manuscript. All authors read and approved the final manuscript.

\section{Ethics approval and consent to participate}

Written informed consent was obtained from all patients prior to publication.

\section{Patient consent for publication}

Patients provided written consent for the publication of their images and associated data.

\section{Competing interests}

Congress participation of the authors AFK and $\mathrm{CH}$ was partially funded by Novocure $\mathrm{GmbH}$. All other authors declare that they have no competing interests.

\section{References}

1. Kreth FW, Thon N, Simon M, Westphal M, Schackert G, Nikkhah G, Hentschel B, Reifenberger G, Pietsch T, Weller M, et al: Gross total but not incomplete resection of glioblastoma prolongs survival in the era of radiochemotherapy. Ann Oncol 24: 3117-3123, 2013.

2. Sanai N, Polley MY, McDermott MW, Parsa AT and Berger MS: An extent of resection threshold for newly diagnosed glioblastoma. J Neurosurg 115: 3-8, 2011.

3. Lacroix M, Abi-Said D, Fourney DR, Gokaslan ZL, Shi W, DeMonte F, Lang FF, McCutcheon IE, Hassenbusch SJ, Holland E, et al: A multivariate analysis of 416 patients with glioblastoma multiforme: Prognosis, extent of resection, and survival. J Neurosurg 95: 190-198, 2001.

4. Tsitlakidis A, Foroglou N, Venetis CA, Patsalas I, Hatzisotiriou A and Selviaridis P: Biopsy versus resection in the management of malignant gliomas: A systematic review and meta-analysis. J Neurosurg 112: 1020-1032, 2010.

5. Oppenlander ME, Wolf AB, Snyder LA, Bina R, Wilson JR, Coons SW, Ashby LS, Brachman D, Nakaji P, Porter RW, et al: An extent of resection threshold for recurrent glioblastoma and its risk for neurological morbidity. J Neurosurg 120: 846-853, 2014.
6. Kirson ED, Dbalý V, Tovarys F, Vymazal J, Soustiel JF, Itzhaki A, Mordechovich D, Steinberg-Shapira S, Gurvich Z, Schneidermann R, et al: Alternating electric fields arrest cell proliferation in animal tumor models and human brain tumors. Proc Natl Acad Sci USA 104: 10152-10157, 2007.

7. Stupp R, Taillibert S, Kanner A, Read W, Steinberg D, Lhermitte B, Toms S, Idbaih A, Ahluwalia MS, Fink K, et al: Effect of tumor-treating fields plus maintenance temozolomide vs. maintenance temozolomide alone on survival in patients with glioblastoma: A Randomized Clinical Trial. JAMA 318: 2306-2316, 2017

8. Ram Z, Kim CY, Nicholas G and Toms S: Compliance and treatment duration predict survival in a phase EF-14 trial of tumor treating fields with temozolomide in patients with newly diagnosed glioblastoma. Neuro Oncol 19 (Suppl 6), 6: vi6-vi7, 2017, https://doi.org/10.1093/neuonc/nox 168.022.

9. Wen PY, Macdonald DR, Reardon DA, Cloughesy TF, Sorensen AG, Galanis E, Degroot J, Wick W, Gilbert MR, Lassman $\mathrm{AB}$, et al: Updated response assessment criteria for high-grade gliomas: Response assessment in neuro-oncology working group. J Clin Oncol 28: 1963-1972, 2010.

10. Stupp R, Mason WP, van den Bent MJ, Weller M, Fisher B, Taphoorn MJ, Belanger K, Brandes AA, Marosi C, Bogdahn U, et al: Radiotherapy plus concomitant and adjuvant temozolomide for glioblastoma. N Engl J Med 352: 987-996, 2005.

11. Taphoorn MJB, Dirven L, Kanner AA, Lavy-Shahaf G, Weinberg U, Taillibert S, Toms SA, Honnorat J, Chen TC, Sroubek J, et al: Influence of treatment with tumor-treating fields on health-related quality of life of patients with newly diagnosed glioblastoma: A secondary analysis of a randomized clinical trial. JAMA Oncol 4: 495-504, 2018.

12. Stupp R, Wong ET, Kanner AA, Steinberg D, Engelhard H, Heidecke V, Kirson ED, Taillibert S, Liebermann F, Dbalý V, et al: NovoTTF-100A versus physician's choice chemotherapy in recurrent glioblastoma: A randomised phase III trial of a novel treatment modality. Eur J Cancer 48: 2192-2202, 2012.

13. Onken J, Staub-Bartelt F, Vajkoczy P and Misch M: Acceptance and compliance of TTFields treatment among high grade glioma patients. J Neurooncol 139: 177-184, 2018.

14. Hemminki K, Liu X, Försti A, Ji J, Sundquist J and Sundquist K: Subsequent brain tumors in patients with autoimmune disease. Neuro Oncol 15: 1142-1150, 2013.

15. Ji J, Shu X, Sundquist K, Sundquist J and Hemminki K: Cancer risk in hospitalised psoriasis patients: A follow-up study in Sweden. Br J Cancer 100: 1499-1502, 2009

16. Wang Y, Li S, Zhang Z, Chen X, You G, Yang P, Yan W, Bao ZS, Yao K. Wang L, et al: Surgical extent impacts the value of the established prognosticators in glioblastoma patients: A prospective translational study in Asia. Head Neck Oncol 4: 80, 2012.

17. Beiko J, Suki D, Hess KR, Fox BD, Cheung V, Cabral M, Shonka N, Gilbert MR, Sawaya R, Prabhu SS, et al: IDH1 mutant malignant astrocytomas are more amenable to surgical resection and have a survival benefit associated with maximal surgical resection. Neuro Oncol 16: 81-91, 2014.

18. Bloch O, Han SJ, Cha S, Sun MZ, Aghi MK, McDermott MW, Berger MS and Parsa AT: Impact of extent of resection for recurrent glioblastoma on overall survival clinical article. J Neurosurg 117: 1032-1038, 2012.

19. Vymazal J and Wong ET: Response patterns of recurrent glioblastomas treated with tumor-treating fields. Semin Oncol 41 (Suppl 6): S14-S24, 2014.

20. Combs SE, Rieken S, Wick W, Abdollahi A, von Deimling A Debus J, Hartmann C: Prognostic significance of IDH-1 and MGMT in patients with glioblastoma: One step forward, and one step back? Radiat Oncol 6: 115, 2011.

21. Millward CP, Brodbelt AR, Haylock B, Zakaria R, Baborie A, Crooks D, Husband D, Shenoy A, Wong $H$ and Jenkinson MD: The impact of MGMT methylation and IDH-1 mutation on long-term outcome for glioblastoma treated with chemoradiotherapy. Acta Neurochir (Wien) 158: 1943-1953, 2016.

22. Yang P, Zhang W, Wang Y, Peng X, Chen B, Qiu X, Li G, Li S, Wu C, Yao K, et al: IDH mutation and MGMT promoter methylation in glioblastoma: Results of a prospective registry. Oncotarget 6: 40896-40906, 2015. 